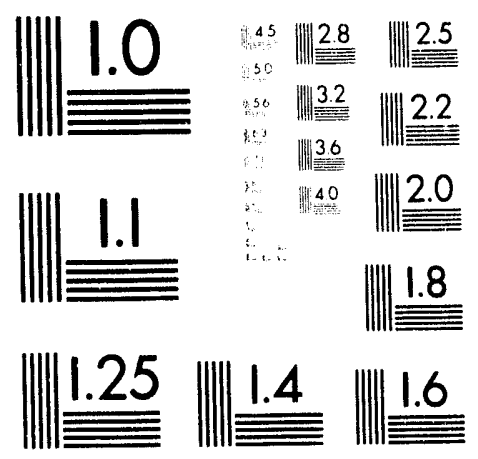



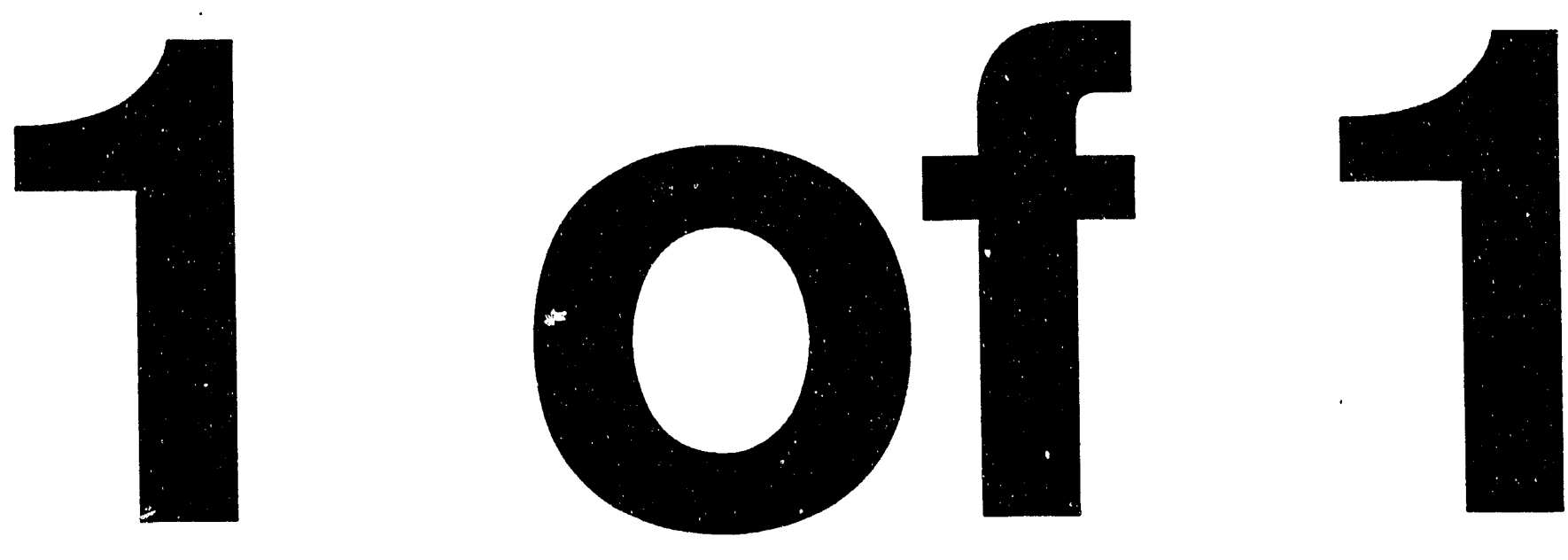
LA-UR $\cdot 93-3072$

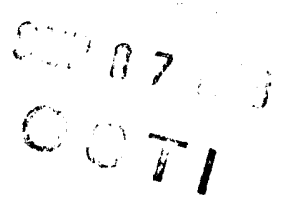

TITLE The accuracy-through-order and the equivalence properties in the algebraic approximant

AUTHOA(S): George A. Baker, Jr.

SUBMirted TO Nonlinear Numerical Methods and Rational Approximation, Conf. Proceedings

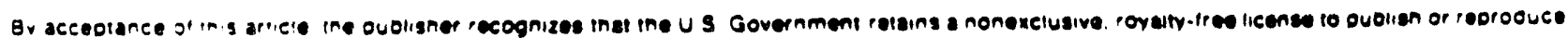




\title{
THE ACCURACY-THROUGH-ORDER AND THE EQUIVALENCE PROPERTIES IN THE ALGEBRAIC APPROXIMANT *
}

\author{
GEORGE A. BAKER, JR. \\ Theoretical Division, Los Alamos National Laboratory \\ University of California, Los Alamos, N. M. 87545 USA
}

\begin{abstract}
In addition to the accuracy-through-order requirement that the defining polynomials not all be divisible by $z$, as required for Padé and integral approximants, there is the further problem of deficiency as pointed out by McInnes. I prove a finite bound on the deficiency and also prove the accuracy-through-order property for algebraic approximants. In addition I prove the equivalence property for algebraic approximants.
\end{abstract}

Key words: Padé approximant, Algebraic approximant, Accuracy-through-order property, Equivalence property

In the study of Pade approximants, there are a number of basic properties, which are fundamental, and are so well known that they are often taken for granted. The theory of algebraic approximants is not so well developed and some of these properties are not yet established. The theory of both types of approximants is a special case of the theory of Hermite-Padé approximants and starts with a linear, polynomial-defining equation,

$$
\sum_{j=0}^{k} P_{j}(z) f_{j}(z)=O\left(z^{s+1}\right),
$$

for the $P_{j}(z)$ 's plus some initial conditions. The approximant involves the solution of

$$
\sum_{j=0}^{k} P_{j}(z) y_{j}(z)=0
$$

where the $y_{j}(z)$ 's are related to each other in a manner akin the the way the $f_{j}(z)$ 's are related to each other. The first basic property is to show that as the degree of contact at the origin in the polynomial defining equation increase indefinitely, then so too does the degree of contact between the $f_{j}(z)$ 's and the $y_{j}(z)$ 's. This property is called the accuracy-through-order property. The next basic property is uniqueness. It consists of two parts. First the uniqueness of the approximant polynomials and second the uniqueness of the solution, given the polynomials, for the approximant. Finally there is the problem of equivalence. It is, in the case of

- Work supported in part by the US Department of Energy (contract W-7405-ENG-36) and in part by the NSF (grant INT-90 13977) 
the Padé approximant stated as, for a function $g(z)$ which is analytic at the origin,

$$
g(z)=\frac{\sum_{j=0}^{l} c_{j} z^{j}}{1+\sum_{j=1}^{m} e_{j} z^{j}}
$$

if and only if,

$$
[L / M]_{g}=[l / m]_{g} \quad \forall L \geq l, M \geq m
$$

where $[\lambda / \mu]_{g}$ is the Padé approximant to $g(z)$ with the degree of the numerator polynomial equal to at most $\lambda$ and the degree of the denominator polynomial equal to at most $\mu$. The Padé polynomials satisfy a defining equation with $s=\lambda+\mu$. The problem of uniqueness of the polynomials has been addressed by Baker and Graves-Morris (1990) and of the solution by McInnes (1992). The Padé approximants also have very useful invariance properties with respect to linear fractional transformations of both their arguments and their values. This property is shared by the algebraic approximants (Baker, 1984). So far however, the accuracy-throughorder property and the equivalence property for algebraic approximants have not been adequately treated. We will give results on these properties.

The algebraic approximants were introduce by Padé (1892) and an investigation of the quadratic case was made by Shafer (1974).

Definition 1. Let a function $f(z)$ be given in terms of its Maclaurin expansion. We define the algebraic polynomials $Q_{j, m}$, by the accuracy-through-order principle by means of

$$
\sum_{j=-1}^{k} Q_{j, m},(z)[f(z)]^{j+1}=O\left(z^{s+1}\right),
$$

where the $Q_{j, m},(z)$ are polynomials of degree at most $m_{j}$ and $s$ is given by

$$
M=\sum_{j=0}^{k}\left(m_{j}+1\right)-1, \quad s=M+m_{-1} .
$$

We will use the convention that if $m_{j}=-1$, then $Q_{j,-1}(z) \equiv 0$. The algebraic approximant is denoted by $\left\langle m_{-1} / m_{0}, \ldots, m_{k}\right\rangle$ and is defined by the solution $y(z)$ of

$$
\sum_{j=-1}^{k} Q_{j, m},(z)[y(z)]^{j+1}=0, \quad \text { where } y(0)=f(0) .
$$

Of course, we can impose additional boundary conditions at $z=0$, if necessary to break the degeneracy of the possible solutions. See McInnes (1992) for an additional discussion of this point.

In order to complete the definition of these approximants, attention must be paid to the possibility that (1) does not uniquely define the polynomials. The points where $Q_{k, m_{k}}(z)$ vanishes correspond to singularities. Consequently, in the case where the polynomials are not uniquely determined it would be desirable, in my view, to eliminate any arbitrary or spurious singularities that are introduced by this lack of uniqueness. We complete these definitions by using the minimal polynomials of Baker and Graves-Morris (1990) 
DEfinition 2. A solution for the algebraic polynomials of type $\left(m_{-1} \ldots m_{k}\right)$ to $f(z)$ is called minimal if it is of the lowest degree in the following sense. First there exists no other solution of type $\left(m_{-1}, \ldots, m_{k}\right)$ for which the actual degree of $Q_{k, m_{k}}$ is smaller. If there exist solutions of type $\left(m_{-1}, \ldots, m_{k}\right)$ for which $Q_{k, m_{k}} \equiv 0$, then we minimize the degree of $Q_{k-1, m_{k-1}}, Q_{k-2, m_{k-2}}$, etc. to find the minimal solution.

Uniqueness is insured by their arguments. In addition, we will impose the condition that $\vec{Q}_{\vec{m}}(0) \neq \overrightarrow{0}$. Baker and Graves-Morris (1994) have proven that we may do so and, although some types $\vec{m}$ may then fail to exist, the table of approximants is as complete, in so far as the derived approximants are concerned, as was the table of approximants derived from the minimal polynomials. Some of the repeats in the table may however have been dropped by this restriction.

A very important property of Pade approximants is that of accuracy-through order. In that case, the requirement th? : the denominator polynomial not vanish at the origin was sufficient to show that the Padé approximant agreed with the defining series to the same order in $z$, as the accuracy of the polynomial defining equations. For integral approximants the same results obtain, but for algebraic approximants, as we shall see, the results are roughly true as well, but the situation is a bit more complex. This question has been addressed by Baker and Graves-Morris (1990) for the case of integral approximants, and by McInnes (1992) in the algebraic case.

In order to analyze the problem of accuracy-through-order for the algebraic approximants, we need, following McInnes (1992), to introduce the concept of deficiency. While our minimal definition for the algebraic polynomials and the results of Baker and Graves-Morris (1994) insure that we need only look at cases for which $\vec{Q}_{\vec{m}}(0) \neq \overrightarrow{0}$, there is another quantity which is important to consider in the algebraic case, i.e., the coefficient of $d y / d z$ in (5) below.

DEFINITION 3. The deficiency $d$ of the algebraic polynomials to $f(z)$ is given by

$$
\sum_{j=0}^{k}(j+1) Q_{j, m},(z)[f(z)]^{j} \underset{z \rightarrow 0}{\propto_{0} z^{d}}
$$

THEOREM 4. If the error in the algebraic polynomial defining equation is $O\left(z^{\mathcal{M}}\right)$ and the deficiency of the algebraic polynomials is $d$, then the algebraic approximant differs from the defining series with an error at worst $O\left(z^{M-d}\right)$.

Proof: First, if we differentiate the approximant defining equation (with error displayed for convenience), we get,

$$
\left(\sum_{j=0}^{k}(j+1) Q_{j, m},(z)[y(z)]^{j}\right) \frac{d y(z)}{d z}=-\sum_{j=-1}^{k} Q_{j, m}^{\prime},(z)[y(z)]^{j+1}+O\left(z^{s+\imath}\right)
$$

where $s$ is defined by (2) and $t$ is the degree of oversatisfaction defined by,

$$
\sum_{j=-1}^{k} Q_{j, m},(z)[f(z)]^{j+1}=O\left(z^{s+1+1}\right)
$$


and where the right hand side is to be of true stated order. Suppose that the deficiency is $d=0$, then by letting $z \rightarrow 0$ in $(5)$ we can compute $y_{1}$ from $y_{0}=f_{0}$, where $y(z)=\sum_{j=0}^{\infty} y_{j} z^{j}$. If we now compute the coefficient of $z^{j}$ in (5) we find that it involves $y_{j}$ linearly with the coefficient proportional to the limit in (4) which is non-zero in this case. Thus we can solve in a unique recursive manner for all the coefficients $y_{j}$. By the defining equation, the $f_{j}$ are also a solution within the error, so we get $y_{j}=f_{j}$ for $j=0, \ldots s+t$ which establishes the accuracy-through-order results for this case. If the deficiency $d>0$, then if we examine (5) since it is also satisfied by $f(z)$ as well as $y(z)$, and $f^{\prime}(0)$ is finite, the left-hand side vanishes like $z^{d}$ and so too must the right-hand side. Note is taken that this step imposes the initial conditions $y^{(j)}(0)=f^{(j)}(0)$ for $j=0, \ldots, d$. Therefore we can divide both sides $i y$ $z^{d}$, which reduces the problem to the case of $d=0$, which we have already treated, except that $O\left(z^{s+t}\right)$ is replaced by $O\left(z^{s+t-d}\right)$, unless $d>s+t$. But it can not be that $d>s$, since we have a minimal solution. For if it were so, then the quantity in (4) multiplied by $f(z)$, would be a different solution set of the same order and the same polynomial degrees of the polynomial defining equations. A linear combination of these two polynomial sets could then be formed which would give a new solution set of order $k-1$ instead of $k$, which is a contradiction to our selection of a minimal set of algebraic polynomials.

In order for the results of the previous theorem to be generally useful. we need to find a limit on the size of the deficiency $d$ which is better than the one just given, $d \leq s$.

THEOREM 5. The deficiency of an essentially unique, minimal algebraic approximant whose polynomials satisfy $\vec{Q}_{\vec{m}}(0) \neq \overrightarrow{0}$ is bounded by

$$
d \leq K k
$$

where $K$ denotes the first non-zero $f_{K}$ after $f_{0}$.

Proof: Let us expand the polynomial defining equation about the given value of $f(0)$. We get,

$$
\sum_{\nu=0}^{k+1} \frac{[f(z)-f(0)]^{\nu}}{\nu !}\left(\sum_{j=\nu-1}^{k} \frac{Q_{j, m},(z)(j+1) ![f(0)]^{j+1-\nu}}{(j+1-\nu) !}\right)=O\left(z^{s+1+1}\right) .
$$

Now we note that the $\nu=0$ term vanishes at $z=0$ as is required by the polynomial defining equations. If the second term does not vanish at $z=0$, then the deficiency is zero. It is the case, when we denote the coefficient of $[f(z)-f(0)]^{\nu}$ by $\mathcal{Q}_{\nu}(z)$, that for at least one $\nu=\mu$ that $\mathcal{Q}_{\mu}(0) \neq 0$. This result can be seen as follows. First consider the case $\nu=k+1$. If $\mathcal{Q}_{k+1}(0) \neq 0$, we have the desired result. Otherwise, consider the case, $\nu=k$. In this circumstance, $\mathcal{Q}_{k}(0)=0$ if and only if $Q_{k-1 m_{k-1}}(0)=0$. We can continue in this manner through all $(k+1 \geq \nu>0)$ the $\mathcal{Q}_{\nu}(0)$ 's. We must eventually find one which does not vanish, since by hypothesis, $\vec{Q}_{m}(0) \neq \overrightarrow{0}$. In order to focus on the computation of the deficiency, it is simplest to consider the determination of $f_{1}$. Let $\mu$ be the smallest value of $\nu$ for which $\mathcal{Q}_{\nu}(0) \neq 0$. From (8) there will be a non-vanishing term, proportional to $f_{1}^{\mu}$, and potentially other terms of order $z^{\mu}$ involving $f_{1}$ but no other series coefficients except $f_{0}$. Let us now examine 
the series in z term by term. The first term (beyond the $\nu=0$ term) is linear in $f_{1}$ and if the coefficient does not vanish, we can solve for $f_{1}$ directly. If it does vanish, then the second term will be a quadratic equation in $f_{1}$ and there will be no mixed terms involving $f_{1}$ or terms involving $f_{j}$ with $j>1$ because their coefficients vanish as a consequence of the vanishing of the first term. The examination continues in this manner so that we either find an equation for $f_{1}$, or the form of the next order term in $z$ involves $f_{1}$ alone. We are however guaranteed that we will find an equation for $f_{1}$ with at least one non-zero coefficient by the $z^{\mu}$ term. It will be of the $\mu$ th order in $f_{1}$ and corresponds to $\mu$ roots which are coincident at the origin. Let $f_{K} z^{K}$ be the first non-zero term in the series expansion of $f(z)-f(0)$. Referring to the definition of deficiency (4) we see that we have established a bound on $d$ in this manner by finding that there is a term of order at most $z^{K \mu}$ with a non-vanishing coefficient. Since $\mu \leq k+1$ the conclusion of the theorem follows as the appropriate power of $f$ in (4) is $\mu-1$. Alternatively, this result can be seen by noting that (4) is equal to the derivative of the form (8) with respect to $f$.

An illustration of these theorems is given by the function

$$
f(z)=-1+z-\frac{1}{2} z^{2}+\frac{3}{8} z^{3}-\frac{5}{16} z^{4}+\frac{35}{128} z^{5}-\frac{63}{256} z^{6}+O\left(z^{7}\right),
$$

which satisfies,

$$
(1+z)[f(z)]^{2}+2(1+z) f(z)+1+z-z^{2}=O\left(z^{7}\right) .
$$

Condition (5) becomes in this case,

$$
2(1+z)(f(z)+1) \frac{d f(z)}{d z}=-[f(z)]^{2}-2 f(z)-1+2 z+O\left(z^{7}\right) .
$$

We see by direct substitution of the defining series in (11) that, as z divides (11), the deficiency is $d=1$ thereby reducing the degree of contact by unity. This deficiency is associated with the the coincidence of two solutions at $z=0$, and the corresponding vanishing of the first two terms in (8). Thus the solution for $f(z)$ is,

$$
f(z)=-1 \pm \sqrt{\frac{z^{2}}{1+z}}+O\left(z^{6}\right) .
$$

We now consider the equivalence properties. In this regard it is useful to define the Beckermann (1990) minimal polynomials. First, we need an ordering relation. If $\vec{m}=\left(m_{-1}, \ldots, m_{k}\right)$ is a vector in the index spact labeling the algebraic polynomials, then the partial ordering relation $\vec{a} \leq \vec{c}$ means that every component of $\vec{a}$ is less than or equal to the corresponding component of $\vec{c}$. The relation $\vec{a}=\vec{c}$ means that every component of the two vectors is equal, and $\vec{a}<\vec{c}$ means that $\vec{a} \leq \vec{c}$ holds but that $\vec{a}=\vec{c}$ fails. Notice that is $a_{i}>c_{i}$ and $a_{j}<c_{j}$ for $i \neq j$, then $\vec{a}$ and $\vec{c}$ are incomparable by these partial ordering relations.

Definition 6. A nontrivial solution of (1) for the algebraic polynomials is called a Beckermann minimal solution if, among all the solutions of (1), there is no other nontrivial solution whose degree is less according to the above given partial ordering relation. 
Notice that a minimal solution according to Definition 2, is also a Beckermann minimal solution, but that there may be Beckermann minimal solutions which are not minimal according to Definition 2. We now give the following results.

THEOREM 7 . The statement $(i) f(z)$ is a functional element at $z=0$ which satisfies

$$
\sum_{j=-1}^{k} Q_{j, m},(z)[f(z)]^{j+1}=0
$$

where $\vec{Q}_{\vec{m}}$ is of true nominal degree and essentially unique, is equivalent to (ii) there exists a functional element $g(z)$ at $z=0$ for which,

$$
\overrightarrow{\mathcal{Q}}_{\vec{M}}=\vec{Q}_{\vec{m}}, \quad \forall \vec{M} \geq \vec{m},
$$

where $\overrightarrow{\mathcal{Q}}_{\vec{M}}$ is a Beckermann minimal solution of type $\vec{M}$ for $g(z)$, the inequality is in the sense of Definition 6 , and $g(z)=f(z)$.

Proof: First, (i) implies (ii) as, if we pick $g(z)=f(z)$, then $\vec{Q}$ is a solution of type $\vec{M}$ for any $\vec{M} \geq \vec{m}$ and as by hypothesis, as $\vec{Q}_{\vec{m}}$ is minimal, it is at least a Beckermann minimal solution of type $\vec{M}$.

Second we must consider, whether (ii) implies (i). Suppose that (ii) holds, therefore we must have

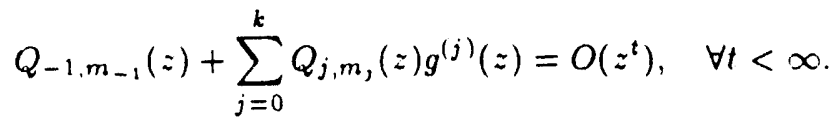

By the Beckermann minimality of $\vec{Q}_{\vec{m}}(z)$, there are no common factors of the type $1+a z$ and the division by any factor of $z^{j}$ leaves (15) unchanged except that $m_{i} \rightarrow$ $m_{i}-j$, as it holds for all $t<\infty$. Since $g(z)$ is a functional element, the left hand side of $(15)$ is regular at $z=0$ and so by (15) must be identically zero. The remaining problem to complete the proof is to show that $g(z)=f(z)$. This result follows from the accuracy-through-order Theorems 4 and 5 and the principle of analytic continuation. The point is that together these theorems show that the degree of contact at the origin between the algebraic approximant and $g(z)$ is infinite, provided that at most $d+1$, where $d$ is the deficiency, of the initial conditions $y^{j}(0)=g^{j}(0)$ hold. This result, by the principle of analytic continuation, proves that any $y(z)=g(z)$ and so $f(z)=g(z)$.

\section{Acknowledgernents}

I wish to thank P. R. Graves-Morris for helpful discussions. 


\section{References}

Baker, George A. Jr.: 1984, 'Invariance properties in Hermite-Padé approximation theory', J. Comp. Es Apl. Math. Vol. no. 11, pp. 49-55

Baker, George A. Jr. and Graves-Morris, P. R.: 1990, Definition and uniqueneas of integral approximants', J. Comp. Et Appl. Maih. Vol. no. 31, pp. 357-372

Baker, George A. Jr. and Graves-Morris, P. R.: 1990, 'Existence of certain sequences of HermutePadé approximants'. J. Comp. Ei Appl. Math. to be published

Beckermann, B.: 1990, "The structure of the singular solution table of the M-Padé approximation problem', J. Comp. E Appl. Math. Vol. no. 32, pp. 3-15

McInnes, A. W.: 1992, 'Existence and uniqueness of algebraic function approximations', Constructive Approximation Vol. no. 8, pp. 1-21

Padé, H.: 1892, 'Sur la répresentation approchée d'une fonction par des fractions rationelles', Ann. de l'Ecole Normale Sup. $3^{i e ́ m e}$ Serie Vol. no. 9, Suppl., pp. 3-93

Shafer, R. E.: 1974. 'On quadratic approximation', SIAM J. Num. Anal. Vol. no. 11. pp. 147-460 

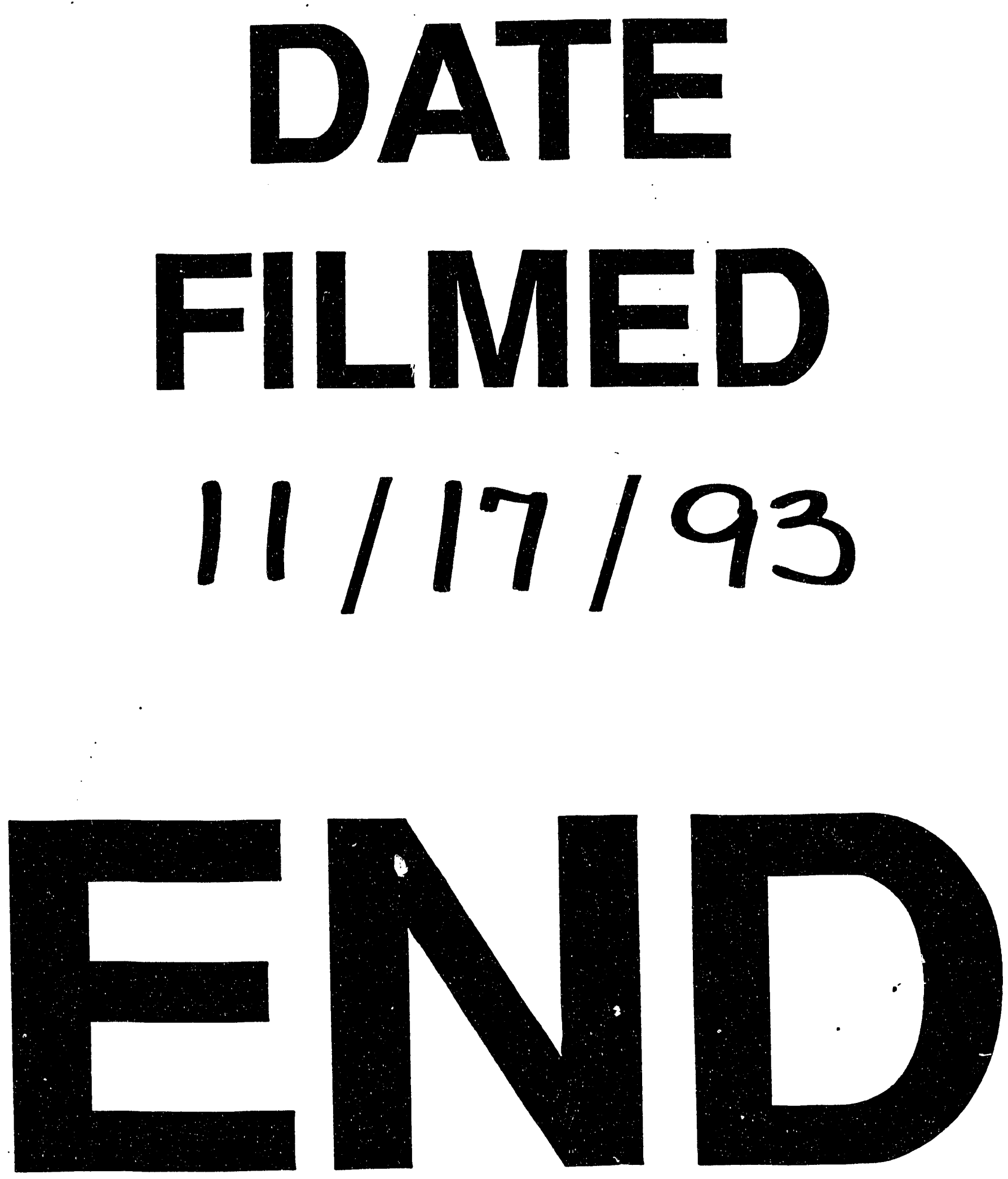
